\title{
Représentations et vécus associés au diabète et à l'éducation thérapeutique :
}

\section{une étude qualitative}

Representations and experiences of diabetes and therapeutic education: a qualitative study

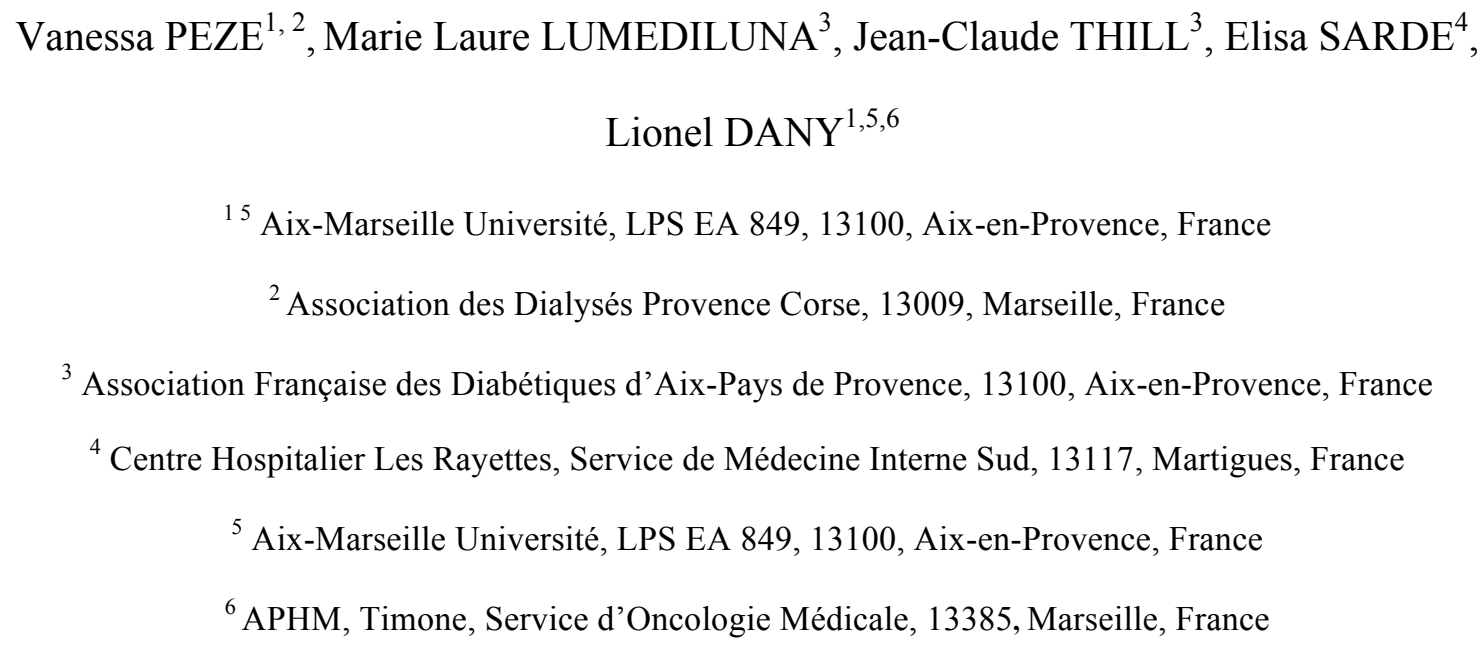

Correspondance : Pr. Lionel DANY, Laboratoire de Psychologie Sociale, Université d'AixMarseille, Maison de la Recherche, 29, avenue Robert-Schuman 13621 Aix-en-Provence cedex 1, France. Courriel : Lionel.Dany@univ-amu.fr Promoteur: Bénévoles, Patients Experts de l'Association Française des Diabétiques d'AixPays-de Provence, France. Marie Laure LUMEDILUNA, Jean-Claude THILL Courriel : afd.aix@gmail.com 
Résumé : Nous avons réalisé une étude qualitative qui avait pour objectif d'appréhender les représentations que les patients associent à l'expérience de leur maladie et à l'éducation thérapeutique (ETP) en les mettant en lien avec leurs caractéristiques socioéconomiques (niveau d'étude, activité professionnelle, revenu) et leur participation à un programme d'ETP. 23 entretiens semi-directifs ont été réalisés auprès de patients atteints de diabète de Type 2 issus de divers milieux socioéconomiques. La moitié des patients a participé à un programme d'ETP. Les entretiens ont été traités à l'aide du logiciel d'analyse textuelle Alceste. Les résultats montrent que les caractéristiques socioéconomiques sont associées de manière significative aux discours portés sur la maladie et l'ETP. En revanche, la variable « participation à un programme » n'est associée à aucune des classes. La manière dont les patients pensent la maladie et l'ETP est étroitement liée au contexte social dans lequel ils sont inscrits. La participation à l'ETP se doit d'être fréquente et inscrite dans la durée pour atténuer les effets de l'inscription sociale des patients.

Mots clés : socioéconomique / représentation / éducation thérapeutique / maladie

Abstract: We realized a qualitative study which aimed at, to apprehend the representations which the patients associate with the experiment of their disease and therapeutic education (ETP) by putting them in link with their socio-economic characteristics (level of study, occupation, income) and their participation in a program of ETP. 23 semi-structured interviews were conducted with patients' diabetics of Type 2 from various socioeconomic backgrounds and half of them participated in a program of ETP. The interviews were treated with the Alceste text analysis software. The results show that the socio-economic characteristics are significantly associated with the speech carried on the disease and the ETP. On the other hand, variable "the participation in a program" is associated with none of the 
classes. The way patients think the disease and the ETP is thus closely related to the social context in which they are registered. The participation in the ETP must be frequent and registered on the duration to attenuate the effects of the social inscription of the patients.

Keywords: socio-economic / representation / therapeutic education / disease

\section{Introduction}

Ces dernières années en France, les programmes d'éducation thérapeutique (ETP) se sont développés de manière importante dans le but d'accompagner les personnes atteintes de maladie chronique dans l'expérience de leur maladie, de réduire la non-observance aux traitements, de limiter l'apparition de complications et d'améliorer la qualité de vie des patients. Parmi ces programmes d'ETP, ceux pour les patients atteints de diabète de type 2 ont vu leurs nombres augmenter. L'objectif est de transmettre aux patients diabétiques, à travers l'ETP, les compétences (compétences d'auto-soins et compétences d'adaptations) qu'ils leur sont nécessaires pour équilibrer leur diabète et éviter l'aggravation de leur état de santé. Le diabète de type 2 (DT2) est une maladie chronique qui demande aux patients de s'impliquer quotidiennement dans leur prise en charge en modifiant leurs habitudes de vie (e.g., adoption d'une alimentation équilibrée, pratique d'une activité physique régulière, astringence à un traitement médical quotidien...). L'acquisition de ces savoirs participe, dans le cadre du diabète, à réguler le taux de glycémie à partir de l'alimentation pour maintenir une qualité de vie favorable.

Des études menées auprès des patients DT2 ayant participé à des programmes d'ETP, qu'ils soient proposés au sein de structures hospitalières ou de villes, ont constaté les effets positifs de ces programmes sur l'observance des patients, révélant une amélioration de leur équilibre glycémique [1]. D'autres travaux ont montré les bénéfices chez ces patients d'un parcours d'ETP personnalisé sur l'acquisition de connaissances sur la maladie et ses traitements [2]. Il 
a été mis évidence que même lorsque l'ETP ne dure qu'une journée, le parcours personnalisé favorise dans la durée l'implication des patients dans leur prise en charge et réduit la détresse qu'ils ressentent pour gérer la maladie [3]. Cependant, on peut observer que les bénéfices reconnus de ces programmes d'ETP ont été évalué auprès de l'ensemble des participants des programmes sans distinction. Les études ne viennent pas discriminer les résultats obtenus en fonction des caractéristiques personnelles et socioéconomiques des patients. Même lorsque les études s'intéressent aux programmes d'ETP personnalisés, elles questionnent principalement la personnalisation des programmes en fonction des besoins des patients et, de façon moindre, la personnalisation des programmes en fonction des caractéristiques socioéconomiques (niveau d'étude, revenu, activités professionnelles) et sociodémographiques (âge, sexe, situation familiale...) et médicales (type de traitements, durée de la maladie...) des patients. Pourtant, la littérature spécialisée en diabétologie identifie largement ces caractéristiques comme des facteurs influençant la prise en charge de la maladie par les patients. L'âge, le sexe, la situation familiale ou encore l'activité professionnelle ont été identifiés comme des obstacles au suivi des prescriptions médicales et hygiéno-diététiques. Les études soulignent qu'un âge avancé rend plus difficile la prise régulière des traitements et que la qualité de vie des patients est altérée par le sexe (féminin), la solitude, une faible activité physique et l'inactivité professionnelle [4-5]. D'autres travaux ont révélé les relations étroites qui existent entre le niveau socioéconomique, le suivi d'une alimentation équilibrée, la pratique physique régulière et l'état de santé chez les patients DT2 [6]. D'autres études ont mis en évidence une relation entre le niveau socioéconomique et l'usage du système de soins (fréquence du suivi médical, consultations de spécialistes, recours aux urgences hospitalières) [7-8], l'équilibre de la glycémie et l'adoption des recommandations hygiéno-diététiques. L'ensemble de ces travaux montre l'impact important du niveau socioéconomique et des caractéristiques personnelles sur la façon dont les patients prennent en charge leur maladie. Pour explorer de 
façon plus discriminante les bénéfices des programmes chez les patients DT2 la prise en considération des caractéristiques sociodémographiques, médicales et socioéconomiques des patients parait alors pertinente. Ces caractéristiques modulant la prise en charge du diabète par les patients, nous faisons l'hypothèse qu'il en est de même pour l'expérience que les patients ont de l'ETP.

Par ailleurs, on peut observer que les recherches qui ont été réalisées sur les bénéfices des programmes d'ETP portent majoritairement sur l'étude des connaissances des patients et moins sur l'étude de leur vécu ou des représentations associés à leur maladie. Pourtant, des données exploratoires récentes ont montré que la perception que les patients ont de la maladie peut être modifiée par ces programmes d'ETP [9]. La littérature en psychologie et les théories sur lesquelles se fonde l'éducation thérapeutique montrent combien les croyances que les patients possèdent sur leur maladie et ses composantes ont une large influence sur la manière dont ils vont s'adapter à la pathologie et ses traitements. Ces croyances peuvent s'imposer comme des freins à leur prise en charge (e.g., difficultés dans le suivi des règles hygiénodiététiques et irrégularité des prises des traitements) ou des leviers (e.g., suivi d'une alimentation équilibrée, pratique régulière d'une activité physique). Les études actuelles sont encore peu nombreuses à prendre en compte les représentations des patients concernant la maladie en fonction de leur participation à un programme d'ETP ou de leurs caractéristiques socioéconomiques [10]. Aborder les croyances des patients sous l'angle des représentations sociales permet « de prendre en considération le rôle de la culture et des valeurs, l'articulation entre connaissances savantes et profanes, le sens que les investissements psychologiques des malades assignent à la gestion des états pathologiques » [11]. Les représentations sociales sur la maladie sont à envisager comme des modalités de connaissances socialement partagées issues simultanément de l'expérience personnelle des patients et de la culture dans laquelle elles s'ancrent, prennent forme et sens, pour expliquer le «monde» de la maladie (e.g., 
causes, importance, attentes, temporalité, ajustements, traitements, ...). Ainsi, ces croyances s'élaborent à partir de l'histoire de vie de la personne et du contexte social et culturel dans lequel elle évolue. Suivant l'appartenance sociale des patients leurs croyances vis-à-vis de la maladie vont être différentes. Les patients partageant des caractéristiques socioéconomiques et/ou personnelles semblables vont partager des croyances et représentations communes sur la maladie et ses composantes. Celles-ci vont guider la manière dont ils vont prendre en charge leur maladie, mais aussi la manière dont ils vont justifier leurs attitudes et leurs comportements vis-à-vis d'elle. De fait, une approche psychosociale compréhensive du vécu de la maladie, prenant en compte le parcours de soin et l'offre d'ETP (vécue ou perçue) en lien avec les représentations sociales de la maladie peut s'avérer heuristique pour comprendre la démarche d'ETP [11,12]. De plus, il semble important de contextualiser cette démarche, par la prise en compte des caractéristiques socioéconomiques, afin de faire part de l'influence de ces caractéristiques sur la régulation de ce vécu et ces représentations. Cette approche permet également d'explorer le lien entre leurs représentations et l'expérience qu'ils font de la maladie et de l'ETP. Cette notion d'expérience vécue, par son ancrage psychosocial et ses liens avec les approches socio-représentationnelles est particulièrement pertinente pour étudier l'expérience de l'ETP, telle que les patients peuvent la mettre en sens. La notion d'expérience vécue comprend deux dimensions : une dimension de l'ordre de l'éprouvé, du vécu (implication psychologique, affectivité, émotions) et une dimension cognitive (expérimentation du monde et sur le monde, construction de la réalité selon des modalités socialement données) [13]. Elle permet de proposer des pistes de réflexion entre les représentations sur la maladie et l'expérience de l'ETP (vécues ou pensées) des patients en lien avec leurs caractéristiques socioéconomiques.

Notre étude avait pour objectif d'explorer les représentations que les patients associent à l'expérience de leur maladie et de l'ETP en lien avec leurs caractéristiques 
sociodémographiques (âge, sexe, situation conjugale), socio-économiques (revenu, niveau d'étude, activité professionnelle) et médicales (dont la participation à un programme d'ETP). Cette étude vise à mieux appréhender la diversité des croyances que les patients possèdent sur la maladie et ses composantes, à éclairer l'effet de la participation à un programme d'ETP sur les croyances des patients, à identifier le lien qui existe entre croyances et caractéristiques sociodémographiques, socioéconomiques et médicales des patients. Ces connaissances permettront de proposer une réflexion autour des bénéficies des programmes d'ETP sur l'expérience de la maladie des patients au vu de leurs caractéristiques socioéconomiques, et de questionner l'impact de ces programmes sur les inégalités sociales de santé.

\section{Population}

Notre recherche a été réalisée auprès de 23 patients DT2 du Centre Hospitalier de Martigues (Tableau I). L'échantillon comprend 12 femmes et 11 hommes, âgés de 38 à 68 ans $(\mathrm{m}=60,9$ ans). La majorité des patients vit en couple. Un patient sur deux a découvert son diabète il y a moins de cinq ans et la plupart est sous antidiabétiques oraux. La majorité des patients est issue de la catégorie socioprofessionnelle des employés et possède un niveau d'étude inférieur au baccalauréat, 8 patients n'ont aucun diplôme et 10 patients ont un diplôme d'études secondaires et d'enseignement professionnel. Plus de sept patients sur dix sont retraités ou sans emploi. Un patient sur cinq dispose d'un revenu total mensuel supérieur à 3100 euros, près de deux sur cinq disposent d'un revenu entre 1500 et 3100 euros, les autres disposants d'un revenu inférieur à 1500 euros.

Au sein de notre échantillon, 13 patients qui ont participé au programme d'ETP « Mieux vivre mon diabète » du service de Médecine Interne Sud du CHU de Martigues et 10 patients n’y ont pas participé. L'ensemble des patients de notre échantillon a déclaré n'avoir jamais participé à un programme d'éducation thérapeutique, une moitié des patients aura participé 
qu'au programme de Martigues. Sur les 11 hommes et 12 femmes qui composent notre échantillon, respectivement 7 hommes et 6 femmes ont participé au programme. Les patients âgés de plus de 60 ans participent majoritairement au programme. Près de trois patients sur quatre qui vit seul à recours au programme tandis que sur l'ensemble des patients en couple la moitié participe au programme. Une majorité des patients qui participent au programme sont des patients retraités. Seulement un patient sur quatre en activité professionnelle participe au programme. 
Tableau I. Description de l'échantillon de patients - Description of the patient sample

\begin{tabular}{|c|c|c|c|c|}
\hline \multirow{2}{*}{\multicolumn{2}{|c|}{ Caractéristiques }} & \multirow{4}{*}{$\begin{array}{c}\begin{array}{c}\text { Total } \\
\text { Patients }\end{array} \\
\begin{array}{c}(\mathbf{n}=\mathbf{2 3}) \\
\mathbf{n}(\mathbf{\%})\end{array} \\
11(48 \%) \\
12(52 \%)\end{array}$} & \multicolumn{2}{|c|}{$\begin{array}{c}\text { Participation au } \\
\text { programme d'ETP }\end{array}$} \\
\hline & & & OUI & NON \\
\hline \multirow{2}{*}{ Sexe } & Homme & & $7(63 \%)$ & $4(36 \%)$ \\
\hline & Femme & & $6(50 \%)$ & $6(50 \%)$ \\
\hline \multirow{3}{*}{ Age } & $<55$ ans & $7(35 \%)$ & $2(29 \%)$ & $5(71 \%)$ \\
\hline & $55-60$ ans & $6(26 \%)$ & $4(50 \%)$ & $2(50 \%)$ \\
\hline & $>60$ ans & $10(39 \%)$ & $7(77 \%)$ & $3(33 \%)$ \\
\hline \multirow{2}{*}{ Situation conjugale } & En couple & $19(83 \%)$ & $10(53 \%)$ & $9(47 \%)$ \\
\hline & Seul(e) & $4(17 \%)$ & $3(75 \%)$ & $1(25 \%)$ \\
\hline \multirow{3}{*}{ Niveau d'études } & Sans diplôme déclaré & $8(35 \%)$ & $4(50 \%)$ & $4(50 \%)$ \\
\hline & Diplôme secondaire (BEP, CAP) & $10(43 \%)$ & $6(50 \%)$ & $4(40 \%)$ \\
\hline & Baccalauréat et plus & $5(22 \%)$ & $3(60 \%)$ & $2(40 \%)$ \\
\hline \multirow{3}{*}{ Situation professionnelle } & Actifs & $5(22 \%)$ & $1(20 \%)$ & $4(80 \%)$ \\
\hline & Inactifs (sans emploi, au foyer) & $5(22 \%)$ & $2(40 \%)$ & $3(60 \%)$ \\
\hline & Retraités & $13(56 \%)$ & $10(77 \%)$ & $3(23 \%)$ \\
\hline \multirow{5}{*}{$\begin{array}{r}\text { Catégories } \\
\text { socioprofessionnelles }\end{array}$} & Artisans, chefs d'entreprise & $5(22 \%)$ & $3(60 \%)$ & $2(40 \%)$ \\
\hline & Professions intermédiaires & $3(13 \%)$ & $3(100 \%)$ & $0(0 \%)$ \\
\hline & Employés & $10(43 \%)$ & $5(50 \%)$ & $5(50 \%)$ \\
\hline & Chômeurs & $2(9 \%)$ & $1(50 \%)$ & $1(50 \%)$ \\
\hline & Au foyer & $3(13 \%)$ & $1(33 \%)$ & $2(67 \%)$ \\
\hline \multirow{3}{*}{$\begin{array}{r}\text { Revenu total mensuel } \\
\text { du ménage }\end{array}$} & $<1500$ euros & $8(35 \%)$ & $4(50 \%)$ & $4(50 \%)$ \\
\hline & Entre 1500 euros et 3100 euros & $10(43 \%)$ & $7(74 \%)$ & $3(36 \%)$ \\
\hline & $>3100$ euros & $5(22 \%)$ & $2(40 \%)$ & $3(60 \%)$ \\
\hline \multirow{3}{*}{ Découverte de la maladie } & $>10$ ans & $9(39 \%)$ & $5(56 \%)$ & $4(44 \%)$ \\
\hline & Entre 5 et 10 ans & $4(17 \%)$ & $4(100 \%)$ & $0(0 \%)$ \\
\hline & $<5$ ans & $10(43 \%)$ & $4(40 \%)$ & $6(60 \%)$ \\
\hline \multirow{4}{*}{ Types traitements } & Cachets & $17(74 \%)$ & $10(59 \%)$ & $7(41 \%)$ \\
\hline & Cachets + injection GPL-1 & $2(9 \%)$ & $0(0 \%)$ & $2(100 \%)$ \\
\hline & Cachets + insuline & $3(13 \%)$ & $2(67 \%)$ & $1(33 \%)$ \\
\hline & Insuline & $1(4 \%)$ & $1(100 \%)$ & $0(0 \%)$ \\
\hline
\end{tabular}


Le programme «Mieux vivre son diabète » se déroule sur quatre semaines à raison d'une journée par semaine (Cf. détails du programme Tableau 2). La première journée permet de faire connaissance avec le patient à partir du diagnostic éducatif et de recueillir des informations sur lui à l'aide d'un questionnaire élaboré pour le programme (e.g., recueils des données sociodémographiques, socioéconomiques et médicales...). Les différentes journées proposent des séances collectives autour de la connaissance de la maladie et de sa gestion médical (e.g connaitre les gestes d'urgence, atelier d'auto-surveillance glycémique), ainsi que des séances collectives autour des règles hygiéno-diététiques et du vécu du diabète (e.g. séance de dessin sur le diabète, « Jeu de sept familles » sur les groupes alimentaires, atelier cuisine, matinée randonnée, séance de relaxation). Le dernier jour, trois mois plus tard, vise à aborder les complications du diabète et à mettre en application les conseils alimentaires dans des simulations de situations de la vie quotidienne (e.g., le choix d'un repas complet au restaurant, la lecture des étiquettes alimentaires des produits régulièrement consommés...). La journée se terminé autour d'une séance sur la gestion du stress et de goûter partagé. Un bilan éducatif est réalisé auprès de chaque patient afin de mesurer les changements déclarés par les patients au cours des deux derniers mois. 
Tableau 2 : Programme d'éducation thérapeutique « Mieux vivre mon diabète » - Therapeutic Education Program « Better Living My Diabetes ».

\begin{tabular}{|c|c|}
\hline Journée 1 (matinée) & Entretien diagnostic éducatif et recueil d'informations \\
\hline \multirow{2}{*}{ Journée 1 (après - midi) } & Qu'est-ce que le diabète? \\
\hline & Expression artistique sur le vécu du diabète \\
\hline \multirow{4}{*}{ Journée 2} & Qu'est-ce que l'équilibre alimentaire? \\
\hline & Préparation culinaire \\
\hline & Jeu de sept familles sur l'équilibre alimentaire \\
\hline & Auto-surveillance glycémique » \\
\hline \multirow{3}{*}{ Journée 3} & Activité physique : promenade en extérieur » \\
\hline & Comment traiter son diabète?» \\
\hline & Connaitre les situation d'urgence et savoir agir » \\
\hline Journée 4 (matinée) & Entretien bilan éducatif \\
\hline \multirow{4}{*}{$\begin{array}{c}\text { Journée } 5 \\
(3 \text { mois après la journée } 4)\end{array}$} & Choisir un menu au restaurant » et « faire ses courses » \\
\hline & Surveillance du traitement et des complications \\
\hline & Découverte d'une séance de relaxation » \\
\hline & Composer un goûter savoureux » \\
\hline
\end{tabular}

\section{Méthode et analyse des données}

Cette étude qualitative s'inscrit dans une démarche compréhensive exploratoire, nous avons réalisé des entretiens de recherche semi-directifs. Ce type d'entretien privilégie l'exploration du vécu par la production d'un discours permettant d'accéder au système de valeurs, de croyances, de représentations du patient. Un guide d'entretien a été élaboré afin d'explorer les représentations et le vécu de la maladie et de l'ETP chez les patients DT2. Le guide d'entretien débutait avec une question large permettant d'ouvrir l'échange sur l'expérience de la maladie par le patient: «Pouvez-vous me parlez de votre diabète?». Suivaient des questions ouvertes permettant de guider les échanges autour de cinq thématiques : la maladie, 
les traitements médicaux, les recommandations hygiéno-diététiques, la prise en charge médicale et l'offre d'ETP.

L'ensemble des entretiens a été traité à l'aide du logiciel d'analyse textuelle Alceste ${ }^{\circledR}$. L'hypothèse générale développée dans le logiciel Alceste ${ }^{\circledR}$ consiste à considérer les lois de distribution du vocabulaire dans les énoncés d'un corpus comme une trace linguistique d'un travail cognitif de reconstruction d'un objet par un individu [14]. Cette méthode met au jour les traces lexicales les plus prégnantes dans le corpus recueilli. Ces dernières, reconstituées sous forme de classes de discours, vont pouvoir être regroupées sous forme de thèmes qui sont autant d'illustrations de la représentation étudiée [15]. Les «classes de discours» (dimensions représentationnelles) sont mises en lien avec les variables étudiées. Le «profil d'une classe » est donné par l'ensemble des mots et des variables les plus significativement associés à une classe de discours, calculé par un coefficient d'association (Chi2 ou $\left.\chi^{2}\right)$. Le logiciel Alceste ${ }^{\odot}$ opère également une Classification Descendante Hiérarchique $(\mathrm{CDH})$ des différentes classes de discours qui permet d'étudier la distribution des classes et de mettre en évidence leurs relations de proximité et d'opposition. Autrement dit, certaines classes sont d'un point de vue sémantique (et thématique) plus proches les unes des autres.

Les résultats de ses analyses vont être présentés dans des tableaux de synthèses. Le tableau III présente les mots typiques de chaque classe de discours, les variables associées et les sousthèmes issus de l'analyse de contenu de ces classes. Le tableau IV concerne les extraits typiques d'entretiens significativement associés aux classes de discours qui concourent à la compréhension des thématiques associées à chacune de ces classes. La figure 1 présente les relations de proximité et d'opposition entre chaque classe. 


\section{Résultats}

L'analyse effectuée à l'aide du logiciel Alceste $^{\odot}$ a mis en évidence sept classes lexicales qui sont autant d'univers de croyances et de comptes rendus d'expériences autour de la maladie et de l'ETP. Nous allons dans un premier temps présenter les caractéristiques de chacune des sept classes en allant de la plus importante classe de discours par rapport au discours total jusqu'à la classe la plus petite. Puis dans un deuxième temps, nous présenterons les liens entre les classes issues de la Classification Descendante Hiérarchique (Figure 1).

\section{L'histoire du diabète : $24 \%$ du discours total - Classe 4}

Cette classe de discours est la plus importante. Les discours portent sur l'histoire du diabète dans le parcours de vie des patients. Les mots les plus significativement associés sont : an (150), mère (98), pied (77), âge (76), mort (67), conséquent (58). Les patients parlent de leur diabète en référence à leur histoire personnelle et familiale, ils évoquent les sentiments qui sont associés à cette expérience. Les propos font part des causes et des conséquences du diabète qu'ils perçoivent. Cette classe de discours est plus typique des artisans et chefs d'entreprises $\left(\chi^{2}=36\right)$ et de ceux qui sont sous traitements par cachets $\left(\chi^{2}=29\right)$.

\section{Le groupe en ETP : $18 \%$ du discours total - Classe 6}

Cet univers souligne l'importance du «groupe » dans la représentation que les patients possèdent de l'ETP. Les termes les plus significativement associés sont : salle (109), groupe (94), parler (91), échange (79), gens (77). Les sous-thèmes montrent que l'ETP est perçue comme étant des séances de formations et/ou de stages où les patients échangent en groupe. Cette classe est plus typique des patients qui sont sous traitement par cachets $\left(\chi^{2}=22\right)$ et qui sont à la retraite $\left(\chi^{2}=16\right)$. 


\section{Le suivi médical : $17 \%$ du discours total - Classe 3}

Les termes les plus significativement associés à cette classe de discours font référence au suivi médical du diabète : diabétologue (390), généraliste (277), spécialiste (144), traiter (118), prise (117), sang (96), rendez-vous (77). Les discours portent sur les principaux médecins (généralistes et diabétologues) qui suivent médicalement les patients. Ils évoquent leurs rôles et leurs fonctions dans la prise en charge médicale des patients. Cette classe est plus spécifique des patients qui ont un revenu compris entre 1500 et 3100 euros $\left(\chi^{2}=49\right)$ et des patients qui ont un diplôme d'études secondaires $\left(\chi^{2}=48\right)$.

\section{Les objectifs de l'ETP : $11 \%$ du discours total - Classe 5}

Cette classe renvoie à la perception de l'ETP en diabétologie. Les mots les plus significativement associés sont : apporter (99), apprendre (90), aider (75), chercher (68), maladie (63), comprendre (58), atelier (57). Les sous-thèmes soulignent que l'ETP est perçue comme une éducation (apport de connaissances, d'informations, ...) des patients sur la maladie et sa gestion. La fonction de l'ETP est de leur apprendre ce qu'est la maladie, de les aider et de discuter avec eux sur le diabète et sa gestion à partir de thématiques précises : la maladie et l'alimentation. Ce discours concerne plus les patients issus de la catégorie socioprofessionnelle des employés $\left(\chi^{2}=38\right)$ et des patients qui sont en activité professionnelle $\left(\chi^{2}=32\right)$.

\section{La forme physique : $11 \%$ du discours total - Classe 1}

Cette classe de discours regroupe les propos des patients qui font référence à leur forme physique et à leur motivation qui apparaissent être des « freins » à bouger plus. On y retrouve principalement les mots : heure (194) ${ }^{1}$, fatigue (164), marcher (103), volonté (86), travail 
(85). Les sous-thèmes concernent le fait d'agir malgré une forme physique et une volonté fragiles. Les notions de temps et de travail apparaissent comme des freins pour l'activité. Ce type de discours est plus typique des patients ayant un revenu mensuel supérieur à 3100 euros $\left(\chi^{2}=99\right)$ et qui ont une activité professionnelle $\left(\chi^{2}=74\right)$.

\section{Le traitement médical : $10 \%$ du discours total - Classe 2}

Cette classe de discours concerne les traitements allopathiques du diabète. Les mots les plus significativement associés sont : cachet (240), prendre (103), piquer (94) matin (87), insuline (76). Les discours évoquent la prise des traitements en appuyant sur la fréquence des prises, leurs effets sur la glycémie et le vécu qui y est associé (habitude, contrainte, ...). Ce discours est plus typique des patients inactifs $\left(\chi^{2}=33\right)$ et qui ont un revenu inférieur à 1500 euros $\left(\chi^{2}=28\right)$

\section{L'alimentation : 9\% du discours total - Classe 7}

Cette classe est la plus petite des classes. Les termes les plus significativement associés à cette classe sont : manger (290), légume (38), viande (24), pâte (26), féculent (25), salade (18). Les patients s'expriment sur leur alimentation depuis qu'ils sont diabétiques. Cette classe est plus typique des retraités $\left(\chi^{2}=82\right)$, des femmes $\left(\chi^{2}=41\right)$, de ceux ayant un revenu inférieur à 1500 euros $\left(\chi^{2}=38\right)$ et des inactifs $\left(\chi^{2}=36\right)$. 
Tableau III. Classes des discours issues de l'analyse ALCESTE ${ }^{\circledR}$ - Classes speeches from the ALCESTE ${ }^{C}$ analysis.

\begin{tabular}{|c|c|c|c|c|c|c|c|}
\hline $\mathrm{N}^{\circ}$ Classe & 1 & 2 & 3 & 4 & 5 & 6 & 7 \\
\hline Thèmes & $\begin{array}{l}\text { FORME } \\
\text { PHYSIQUE }\end{array}$ & $\begin{array}{l}\text { TRAITEMENT } \\
\text { MEDICAL }\end{array}$ & $\begin{array}{c}\text { SUIVI } \\
\text { MEDICAL }\end{array}$ & $\begin{array}{l}\text { HISTOIRE DU } \\
\text { DIABETE }\end{array}$ & $\begin{array}{l}\text { OBJECTIFS } \\
\text { ETP }\end{array}$ & $\begin{array}{l}\text { GROUPE } \\
\text { ETP }\end{array}$ & L'ALIMENTATION \\
\hline $\begin{array}{l}\% \text { du discours } \\
\text { total }\end{array}$ & $11 \%$ & $10 \%$ & $17 \%$ & $24 \%$ & $11 \%$ & $18 \%$ & $9 \%$ \\
\hline $\begin{array}{l}\text { Variables } \\
\text { associées }\end{array}$ & $\begin{array}{c}>3100 \text { euros } \\
\text { Actifs }\end{array}$ & $\begin{aligned} & \text { Inactifs } \\
< & 1500 \text { euros }\end{aligned}$ & $\begin{array}{c}1500 \text { à } 3100 \text { euros } \\
\text { BEP, CAP ... }\end{array}$ & $\begin{array}{c}\text { Artisans, chefs } \\
\text { Cachets et injection }\end{array}$ & $\begin{array}{l}\text { Employés } \\
\text { Actifs }\end{array}$ & $\begin{array}{l}\text { Cachets } \\
\text { Retraités }\end{array}$ & $\begin{array}{c}\text { Retraités } \\
\text { Femmes } \\
\text { Inactifs } \\
<1500 \text { euros }\end{array}$ \\
\hline $\begin{array}{c}\text { Termes les } \\
\text { plus associés }\end{array}$ & $\begin{array}{l}\text { Heure, travail, } \\
\text { fatigue, midi, } \\
\text { rentrer, marcher, } \\
\text { volonté. }\end{array}$ & $\begin{array}{l}\text { Cachet, bas, } \\
\text { Prendre, piquer, } \\
\text { matin, insuline, } \\
\text { piqure, baisse. }\end{array}$ & $\begin{array}{l}\text { Diabétologue, } \\
\text { généraliste, } \\
\text { docteur, médecin, } \\
\text { spécialiste, traité. }\end{array}$ & $\begin{array}{l}\text { An, mère, pied, } \\
\text { age, mort, } \\
\text { conséquent. }\end{array}$ & $\begin{array}{c}\text { Apporter, } \\
\text { apprendre, } \\
\text { aider, chercher, } \\
\text { maladie, atelier, } \\
\text { comprendre. }\end{array}$ & $\begin{array}{l}\text { Salle, groupe, } \\
\text { parler, échange, } \\
\text { gens, réunion. }\end{array}$ & $\begin{array}{l}\text { Manger, légume, } \\
\text { viande, pâte, } \\
\text { féculent, salade. }\end{array}$ \\
\hline \multirow{3}{*}{ Sous-thèmes } & $\begin{array}{l}\text { AGIR } \\
\text { Marcher, lever, } \\
\text { rentrer, sortir, } \\
\text { aller. }\end{array}$ & $\begin{array}{l}\text { TEMPORALITE } \\
\text { Matin, midi, soir, } \\
\text { régulier, jour. }\end{array}$ & $\begin{array}{l}\text { PROFESSIONNELS } \\
\text { DE SANTE } \\
\text { Diabétologue, } \\
\text { généraliste, } \\
\text { spécialiste, médecin, } \\
\text { docteur. } \\
\end{array}$ & $\begin{array}{l}\text { FAMILLE } \\
\text { Mère, père, grand- } \\
\text { père, sœur, grand- } \\
\text { mère, enfant. }\end{array}$ & $\begin{array}{l}\text { FONCTIONS } \\
\text { Lire, apporter, } \\
\text { apprendre, aider, } \\
\text { chercher, discuter, } \\
\text { comprendre, }\end{array}$ & $\begin{array}{l}\text { APPELLATION } \\
\text { Réunion, stage, } \\
\text { formation, atelier. }\end{array}$ & $\begin{array}{c}\text { ALIMENTS } \\
\text { Légumes, viande, pâte, } \\
\text { féculent, salade, fruit, } \\
\text { tomate, poisson, pomme }\end{array}$ \\
\hline & $\begin{array}{l}\text { VOLONTE } \\
\text { Fatigue, force, } \\
\text { énergie, volonté, } \\
\text { battre, laisser. }\end{array}$ & $\begin{array}{c}\text { VECU } \\
\text { Piquer, prendre, } \\
\text { obliger, } \\
\text { contraindre, } \\
\text { habitude, sérieux, } \\
\text { oublier. }\end{array}$ & $\begin{array}{c}\text { ROLES ET } \\
\text { FONCTIONS } \\
\text { Traiter, envoyer, } \\
\text { suivre, prescrire, } \\
\text { orienter, surveiller. }\end{array}$ & $\begin{array}{l}\text { CAUSES ET } \\
\text { CONSEQUENCES } \\
\text { Hérédité, âge, } \\
\text { sédentarité, } \\
\text { surpoids, pieds, } \\
\text { jambe, mort, } \\
\text { mourir, couper. }\end{array}$ & $\begin{array}{l}\text { EDUCATION } \\
\text { Connaissance, } \\
\text { information, } \\
\text { éducation, } \\
\text { savoir, cours, } \\
\text { atelier. }\end{array}$ & $\begin{array}{l}\text { GROUPE } \\
\text { Ensemble, } \\
\text { humain, gens, } \\
\text { monde, groupe, } \\
\text { malade. }\end{array}$ & $\begin{array}{c}\text { ACTIONS } \\
\text { Manger, cuire, boire, } \\
\text { sucrer, grignoter, } \\
\text { diminuer, redescendre. }\end{array}$ \\
\hline & $\begin{array}{l}\text { TEMPORALITE } \\
\text { Heure, matin, } \\
\text { journée, semaine, } \\
\text { nuit. }\end{array}$ & $\begin{array}{l}\text { VARIATIONS DE } \\
\text { LA GLYCEMIE } \\
\text { Descendre, } \\
\text { remonter, baisser, } \\
\text { stabiliser. }\end{array}$ & & $\begin{array}{c}\text { VECU } \\
\text { Attention, } \\
\text { conscient, peur, } \\
\text { craindre, frustrer. }\end{array}$ & $\begin{array}{l}\text { THEMATIQUES } \\
\text { Maladie, santé, } \\
\text { aliment, diététique, } \\
\text { nourriture. }\end{array}$ & $\begin{array}{l}\text { ECHANGES } \\
\text { Parler, trouver, } \\
\text { échanger. }\end{array}$ & \\
\hline
\end{tabular}


Tableau IV. Extraits d'entretiens typiques pour chaque classe de discours - Excerpts from interviews typical for each speech class.

\begin{tabular}{|c|c|}
\hline \multirow{2}{*}{$\begin{array}{l}\text { CLASSE } 1 \\
\text { Forme } \\
\text { physique }\end{array}$} & $\begin{array}{l}\text { "C'est juste aller marcher l'après-midi comme il faut, mais après mon travail je n'y arrive } \\
\text { plus. Est-ce-que c'est parce que je manque d'énergie...Je sens que je suis vraiment fatiguée } \\
\text { ce n'est pas...» }\end{array}$ \\
\hline & $\begin{array}{l}\text { "C'est vrai que l'on me dit que vous n'avez pas besoin de faire une activité sportive, mais } \\
\text { ce qu'on vous demande c'est de sortir marcher pendant une heure, de bien marcher pendant } \\
\text { une heure, et c'est vrai que pour l'instant je n'ai pas cette volonté-là. » }\end{array}$ \\
\hline \multirow{2}{*}{$\begin{array}{l}\text { CLASSE } 2 \\
\text { Traitement } \\
\text { médical }\end{array}$} & $\begin{array}{l}\text { "Le médicament, je le prends matin, midi et soir. C'est celui que je dois prendre } \\
\text { sérieusement. J'en prends deux, le matin, le midi et le soir, et puis je me fais une injection le } \\
\text { soir avant de souper.» }\end{array}$ \\
\hline & $\begin{array}{l}\text { « Mais, ce n'est pas bien ça, il ne faut pas agir comme j'agis parce que le diabète, il monte, } \\
\text { il descend, il ne se régularise pas quand c'est comme ça. C'est pour ça qu'on vous dit que le } \\
\text { traitement, il faut le prendre matin, midi et soir. » }\end{array}$ \\
\hline \multirow{2}{*}{$\begin{array}{l}\text { CLASSE } 3 \\
\text { Suivi } \\
\text { médical }\end{array}$} & $\begin{array}{l}\text { «Je suis suivie à l'hôpital X par le diabétologue, et c'est mon médecin traitant le docteur X } \\
\text { qui m'a orienté vers le centre médical cardio vasculaire et qui me suis pour mon diabète } \\
\text { également. Il me prescrit l'ordonnance. » }\end{array}$ \\
\hline & $\begin{array}{l}\text { «Je viens ici, pour la visite avec le diabétologue, il me prescrit des prises de sang, et après, } \\
\text { je vais chez mon médecin traitant. » }\end{array}$ \\
\hline \multirow{2}{*}{$\begin{array}{l}\text { CLASSE } 4 \\
\text { Histoire du } \\
\text { diabète }\end{array}$} & $\begin{array}{l}\text { "Moi si cela se passe bien, si je n'ai pas de complications, c'est parce que je fais } \\
\text { attention parce que quand je vois comment cela s'est passé avec mon frère... Lui, il ne } \\
\text { faisait pas attention, lui, il a eu des complications, des conséquences très, très graves, on a } \\
\text { dû lui couper une jambe. » }\end{array}$ \\
\hline & $\begin{array}{l}\text { "Oui, de toute façon, moi, c'est un problème de famille le poids. Moi, ma mère, elle a } \\
\text { jamais fait attention à son poids, elle était grosse, elle était maigre, mais alors cela n'a jamais } \\
\text { été un problème donc le diabète c'est ça aussi...» }\end{array}$ \\
\hline \multirow{2}{*}{$\begin{array}{l}\text { CLASSE } 5 \\
\text { Objectifs } \\
\text { ETP }\end{array}$} & $\begin{array}{l}\text { "Mener les connaissances sur la pathologie et puis apporter les éléments pour, si ce n'est } \\
\text { faire disparaitre, mais en tout cas éviter les aggravations dues au diabète. Donc, c'est ce qui } \\
\text { est éducation à la santé liée à la pathologie, peut-être les règles hygiéno-diététiques aussi, et } \\
\text { je trouve que les ateliers qui ont été faits ont apporté des éléments positifs là-dessus. » }\end{array}$ \\
\hline & $\begin{array}{l}\text { «Pour apprendre, vous ne savez pas tous, au niveau de la nourriture, au niveau des glucides, } \\
\text { tout ça, donc c'est pour apprendre, pour apprendre quelque chose sur la maladie et surtout } \\
\text { sur la nourriture.» }\end{array}$ \\
\hline \multirow{2}{*}{$\begin{array}{l}\text { CLASSE } 6 \\
\text { Groupe } \\
\text { ETP }\end{array}$} & $\begin{array}{l}\text { "Des explications et des questions réponses. Cela voulait dire qu'il y avait un échange. } \\
\text { C'est important dans la mesure où la personne qui pose une question a une réponse, que ce } \\
\text { soit un patient qui pose une question au médecin et il a sa réponse, ou que ce soit le médecin } \\
\text { qui pose la question au patient et il a sa réponse. » }\end{array}$ \\
\hline & $\begin{array}{l}\text { "C'est ce qui doit tourner autour du stage, les échanges de malades, chacun doit dire } \\
\text { comment il a fait, comme aux alcooliques anonymes avec la partie psychologie, relation de } \\
\text { groupe, tout ça.» }\end{array}$ \\
\hline \multirow{2}{*}{$\begin{array}{c}\text { CLASSE } 7 \\
\text { L'alimentation }\end{array}$} & $\begin{array}{l}\text { "Le féculent, c'est } 30 \mathrm{~g} \text { sec pour les pâtes par exemple, un peu de fromage et un fruit. Si je } \\
\text { ne prends pas de fromage, je prends un yaourt, et ça midi et soir, le matin un bout de pain, } \\
\text { comme ça, et puis une petite tranche le midi. » }\end{array}$ \\
\hline & $\begin{array}{l}\text { «D'ailleurs l'huile d'olive, on la verse à la cuillère maintenant alors qu'avant on mettait ça } \\
\text { dans le plat, on la versait comme ça, sucre lent, viande, poisson, je mange beaucoup de } \\
\text { poissons bouillis, au cours bouillon quoi, et un fruit. » }\end{array}$ \\
\hline
\end{tabular}




\section{Liens entre les classes}

Les résultats indiquent plusieurs partitions (Figure 1). La première partition (diabète / alimentation) montre que les discours des patients autour de la maladie et de l'éducation thérapeutique donnent une place singulière à l'alimentation dans leur vécu. La classe de discours portant sur l'alimentation se détache des autres discours abordés sur l'expérience de la maladie et sur l'ETP. La seconde partition marque une scission entre les discours portant sur la gestion par le patient de la maladie (gestion personnelle) et ceux portant sur la gestion par les soignants de la maladie (gestion médicale). La troisième partition dissocie le suivi médical de l'éducation thérapeutique. Les discours portant sur 1'ETP apparaissent intégrés dans la prise en charge médicale de la maladie et sont marqués par la quatrième partition (objectif ETP et groupe ETP). La cinquième partition sépare les discours portant sur l'histoire de la maladie et ceux sur les moyens de la gérer, pour laquelle les discours sur l'activité physique et les traitements médicaux sont regroupés.

Figure 1. Classification descendante hiérarchique des classes lexicales et répartition des thématiques générales - Hierarchical Ascendant Classification of the lexical classes and organization of the general thematics

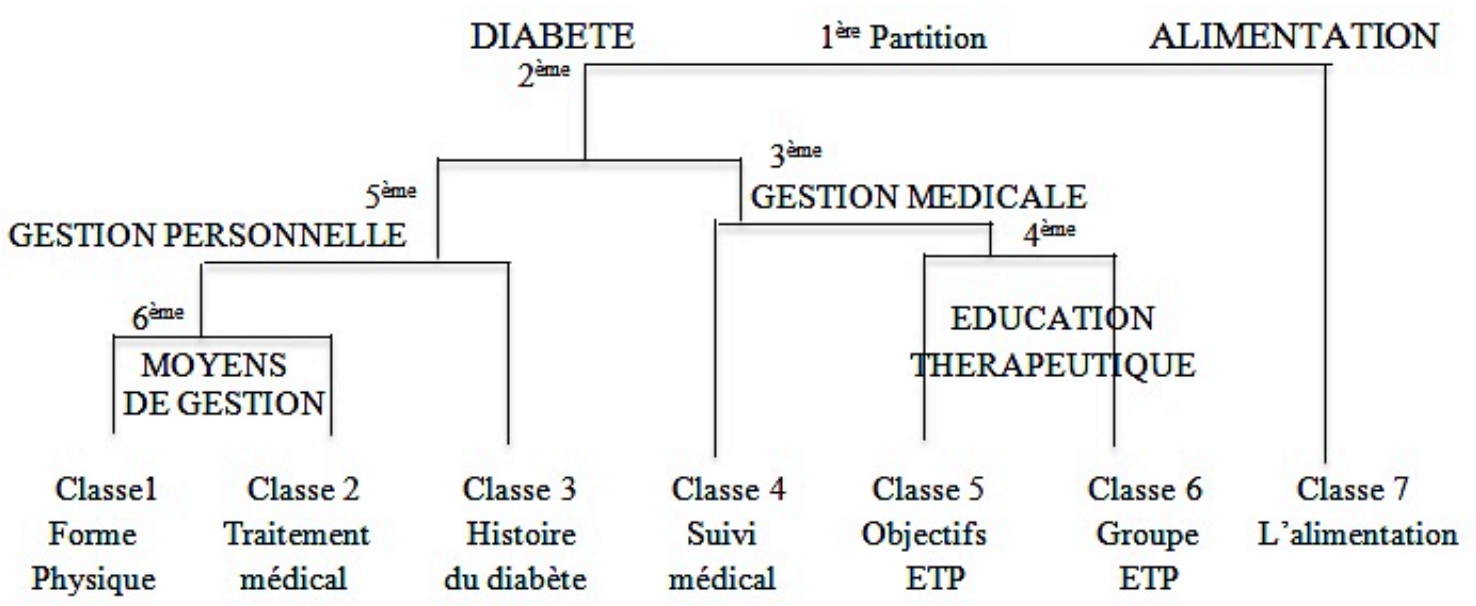




\section{Discussion}

L'analyse des entretiens a permis la mise à jour de plusieurs classes lexicales qui rendent compte de focalisations diverses des patients sur la maladie et sur l'éducation thérapeutique en lien avec leurs caractéristiques sociodémographiques, médicales et socioéconomiques. Ces résultats attestent de la régulation socio-contextuelle des représentations sociales de la maladie, de son vécu et de l'ETP, et spécifient l'ancrage psychosociologique de ces représentations [16]. La participation et l'inscription sociale des patients (définis ici à partir de leurs caractéristiques socio-économiques) contribuent tout particulièrement à la valorisation de dimensions représentationnelles communes de la maladie et de l'ETP. Autrement dit, le rapport qu'entretiennent ces patients avec «les objets représentés » (l'ETP et la maladie) est étroitement lié au contexte social dans lequel ils sont inscrits. Nous allons, dans un premier temps, discuter de l'association de ces variables à certaines composantes importantes du discours produit sur la maladie et l'expérience du diabète pour penser l'offre d'ETP. Dans un deuxième temps, nous discuterons l'absence de lien entre la variable «participation à un programme d'ETP» et les contenus représentationnels spécifiques de l'ETP. Enfin, nous proposerons des pistes de réflexion autour de la régulation de l'expérience de l'ETP au regard des variables socioéconomiques. Il est nécessaire de souligner certaines limites de cette étude. Du fait des caractéristiques de l'échantillon, de la méthode et parce que l'étude est monocentrique, les données ne sont pas généralisables. Les analyses produites ont davantage vocation à la transférabilité. 


\section{Associations des variables (sociodémographique, médicale, socioéconomique) à certaines composantes du discours}

Parmi les composantes représentationnelles importantes du discours des patients, on peut évoquer l'activité physique, les traitements, l'alimentation et, plus globalement, l'expérience du diabète. Les résultats montrent que sur l'ensemble des caractéristiques socioéconomiques qui ont été retenues dans notre étude, trois variables (le revenu, la catégorie socioprofessionnelle et la situation professionnelle) sont associées de manière significative à des classes de discours. On observe que les patients en activité et ceux ayant un revenu plus élevé évoquent davantage que les autres patients leur «forme physique ». Les patients qui sont sans activité professionnelle et qui ont un revenu moindre parlent eux de façon plus significative du traitement médical et de leur alimentation. Lorsque les patients les plus aisés évoquent la «forme physique », ils parlent de leurs difficultés à bouger au quotidien. La présence de ce discours sur la « forme physique », comme moyen de gestion de la maladie au sein d'une classe portant sur la gestion personnelle de la maladie met en évidence l'importance de l'activité physique pour ces patients afin d'intervenir par eux-mêmes sur le diabète. Ce résultat, en rappelant le lien entre niveau d'étude et revenu, peut être mis en relation avec les études qui soulignent que la pratique d'une activité physique est considérée par les personnes diplômées comme bénéfique pour maintenir un «poids de forme» par rapport aux personnes non diplômées [17]. En outre, les patients diabétiques issus d'un milieu socioéconomique fragile ont un niveau d'activité physique qui se situe en dessous des recommandations contrairement aux patients qui sont issues d'un milieu plus aisé [18]. Il semble donc que les patients DT2 issus d'un milieu socioéconomique plus aisé s'approprient davantage les recommandations sur la pratique d'une activité physique régulière que les autres patients car elles sont plus proches de leurs comportements de santé quotidien, 
comportements qui s'inscrivent dans une pratique de prévention et de maintien d'un état de santé favorable.

Une autre dimension importante concerne les traitements. On observe que les discours produits sur les traitements sont plus associés aux patients les plus défavorisés (inactifs et revenu moindre). Leurs discours sur les traitements dénotent la démarche curative dans laquelle semble s'inscrire les patients issus des milieux plus défavorisés pour traiter leur diabète par opposition à la démarche préventive des patients DT2 observés ci-dessus. Les études soulignent que l'on observe moins de comportements de prévention (e.g., adoption d'une alimentation équilibrée, pratique d'une activité physique régulière, surveillance médicale, ...) chez les personnes défavorisées [19]. L'attention que ces personnes portent au soin de leur corps est moins importante que chez les autres. Leur corps est davantage perçu comme un outil fonctionnel qui permet de réaliser et maintenir les tâches de la vie quotidienne [20]. Ces constats peuvent expliquer que les discours des patients DT2 les plus défavorisés soient, de façon plus marquée, portés sur les traitements médicaux et moins sur la pratique d'une activité physique. Les discours sur les traitements soulignent que cette démarche curative serait associée à la prescription médicale. Les patients laissent entrevoir la confiance qu'ils accordent au médecin pour traiter la maladie. Ces discours questionnent le rôle que ces patients accordent à la sphère médicale (médecin traitant et diabétologue) pour traiter leur diabète comparativement aux autres patients pour lesquels une part importante du traitement du diabète semble associée à l'hygiène de vie et in fine à la responsabilité personnelle. Des études soulignent que dans les milieux les plus défavorisés «l'image du médecin reste confondue à celle de l'autorité en général » [21]. Par conséquent, nous posons l'hypothèse que la « soumission » au système médical et l'appréhension du corps comme un outil fonctionnel inscrit les patients dans une démarche curative de leur maladie et les conduit 
à remettre le traitement de leur corps entre les mains des médecins. Ils s'inscrivent dans une position plus passive dans la prise en charge de leur maladie et de ses soins comparativement aux autres patients qui semblent adopter des changements dans leurs hygiènes de vie. Les patients aisés, dans leur «souci» et attention portés à leur corps, vont s'inscrire dans une démarche plus active, par l'adoption de comportements de prévention (e.g., la pratique d'une activité physique) visant à contrôler les complications du diabète. Ce rapport préalable au souci de soi peut expliquer leur adhésion aux recommandations hygiéno-diététiques. Il semble donc que les pratiques alimentaires et sportives soient inscrites dans les habitudes des patients les plus aisées indépendamment de leur maladie, et que les recommandations liées à la maladie ne feraient qu'accentuer ces pratiques. En outre, on observe que les discours portant sur l'alimentation ne se situent pas dans les discours portant sur la gestion personnelle de la maladie. L'alimentation dépasse le strict cadre du «traitement » de la maladie et s'inscrit dans une visée plus large.

Des études antérieures ont montré qu'il existe des différences dans la manière de se représenter l'alimentation et de s'alimenter en fonction du niveau socioéconomique [22]. Au sein des milieux défavorisés certains aliments sont préférés en raison du caractère symbolique auxquels ils sont associés (e.g., la viande associée à la force et l'énergie) et c'est la quantité alimentaire qui peut être valorisée à l'inverse des milieux plus aisés qui s'intéressent davantage à la qualité des aliments qu'ils consomment [23]. Dans les milieux aisés, une grande attention est portée à l'alimentation dans un souci de santé, les femmes diplômées prêtent un grand intérêt au suivi des règles diététiques recommandées pour le maintien de leur santé et de leur ligne [24]. A l'inverse, pour les femmes ayant un faible revenu et sans emploi, l'attention est centrée sur les tâches domestiques et familiales, le suivi des règles diététiques passant au second plan [25]. Ces observations soulignent que les normes de corpulence et les règles diététiques s'éloignent des préoccupations des femmes à mesure que l'on descend dans 
la hiérarchie sociale [26]. Ces normes prennent plus d'importance dès lors que les femmes de milieux défavorisés se rapprochent des milieux plus aisés au travers du monde du travail [26,27]. Ces constats expliquent le lien entre les variables (genre, revenu faible et inactivité) et les discours élaborés sur l'alimentation. Pour les personnes issues de milieux défavorisés, l'alimentation peut être appréhendée comme un élément de consommation qui permet leur intégration sociale [28,29]. En opposition, l'alimentation dans les milieux aisés est mise en relation directe avec la santé et la minceur, allant même jusqu'à venir moduler les goûts et les préférences des plus diplômés [26]. Ainsi, le lien perçu entre alimentation et santé est « intimement liée à la position dans la structure sociale » [30]. Ces éléments soulignent l'investissement socio-symbolique différent de l'alimentation en fonction du contexte social et peuvent expliquer, dans une certaine mesure, que l'on observe chez les patients issus de milieux les plus fragiles un faible suivi des recommandations alimentaires [31]. Ces constats formulent également des éléments d'explications sur la place singulière que prend l'alimentation pour l'ensemble des patients dans le discours qu'ils portent sur l'expérience de leur maladie. Nos constats sur l'attention que les personnes aisées portent au suivi d'une « alimentation santé » dans leur quotidien appuient l'hypothèse que les patients DT2 aisés ont intégré dans leur quotidien indépendamment de leur maladie, l'alimentation comme objet de maintien et/ou de prévention de la santé, les recommandations alimentaires prescrites dans le cadre du diabète venant s'ajouter à leurs pratiques quotidiennes. Ce faisant, les changements d'habitudes alimentaires auxquels les patients doivent procéder pour équilibrer leur diabète s'inscrivent dans des conduites alimentaires plus larges que celles découlant du contexte de leur maladie et s'inscrivent d'autant plus facilement qu'elles sont en cohérence avec le lien que perçoivent les patients DT2 entre alimentation et santé. Autrement dit, le lien entre alimentation, santé et caractéristiques socioéconomique transcende de fait le seul contexte de la maladie pour signifier l'inscription sociale des patients. 
Les discours tenus sur l'histoire de la maladie prennent une part importante dans le discours total des patients. Les patients établissent un lien entre les «membres de leurs familles », les « causes et conséquences » du diabète et les craintes qu'ils éprouvent vis-à-vis des complications. La répartition des classes (cf. Figure 1) montre que les discours tenus sur la gestion personnelle de la maladie sont associés aux discours relevant de l'histoire de la maladie. L'expérience que les patients ont du diabète au cours de leur vie s'élabore au travers de leur contexte familial et semble interagir avec la manière dont ils vivent et gèrent leur maladie. La notion d'expérience vécue trouve ici sa pertinence, car elle postule que l'expérience engage des dimensions cognitives et émotionnelles et qu'elle est étroitement liée avec le contexte social et culturel où vivent les individus [14]. L'expérience produite dans la rencontre intersubjective est sociale et socialement construite. Les représentations partagées par les patients interviennent dans la mise en forme de leur vécu de la maladie. Par exemple, la gravité perçue de la maladie préexiste le plus souvent à l'expérience de la maladie par les patients. On comprend alors que certaines études ont pu observer que les patients qui expriment des réticences lors du passage au traitement par insuline sont plus fréquemment des patients qui ont des membres de leurs familles qui ont eu un diabète qui s'est compliqué [32]. Dans notre étude, les patients qui sont traités simultanément par cachets et par injections évoquent de façon plus importante l'histoire de leur diabète. Ils évoquent la gravité perçue de leur maladie, les conséquences perçues du diabète chez les membres de leur famille et expriment leurs sentiments vis-à-vis de la maladie et de ses complications. Les études réalisées sur la perception de l'insuline chez les patients DT2 mettent en évidence que les patients encore sous cachets perçoivent la mise sous insuline comme « le signe que la maladie s'est aggravée » $[33,34]$. On peut alors faire l'hypothèse que les patients, sous cachets et 
injections, en évoquant l'histoire de leur maladie expriment les craintes qu'ils éprouvent visà-vis du passage à l'insuline.

\section{Absence de lien entre la variable "participation à un programme d'ETP » et les contenus représentationnels (objectifs ETP et groupe ETP) de l'ETP.}

Un autre résultat important concerne le fait que la variable «participation à un programme d'ETP » ne soit pas associée aux classes de discours issues de l'analyse Alceste. Autrement dit, la participation à un programme d'ETP ne conduirait pas, pour notre échantillon, à l'expression d'un discours spécifique sur l'expérience de la maladie mais aussi sur l'ETP. Ce résultat a priori étonnant nécessite d'être discuté car il interroge la modification potentielle ou possible des représentations (sur la maladie, les soins ou autre) à partir d'un dispositif éducatif.

Le premier élément de discussion porte sur l'expression d'un discours sur l'ETP qui n'est pas spécifiquement liée aux patients qui ont participé au programme. Quelle que soit la participation des patients à un programme, l'univers représentationnel de l'ETP est marqué par la dimension éducative et groupale de l'ETP, dimension perçue comme intégrée à la prise en charge médicale mais aussi perçue comme une alternative à cette dernière pour certains patients. L'ETP est décrite comme un dispositif autant éducatif qu'interactif qui aide à mieux gérer la pathologie par l'acquisition de connaissances, notamment sur l'alimentation. Elle forme une réponse aux questions que les patients se posent à un moment donné de leur parcours thérapeutique et s'intègre dans la prise en charge médicale tout en proposant aux patients une alternative au suivi médical traditionnel. La littérature spécialisée sur les représentations sociales insiste sur la stabilité des contenus représentationnels et sur leur résistance au changement [35]. Leurs changements impliquent certaines conditions, en 
particulier, la mise en œuvre de nouvelles pratiques et le fait que la situation de changement qui accompagne ces nouvelles pratiques soit considérée comme irréversible [36]. On peut noter que les patients de notre échantillon qui témoignent de leur expérience du programme d'ETP n'ont participé qu'une seule fois au programme. Ces constats marquent que la présence d'interactions succinctes et ponctuelles entre les patients et les intervenants du programme peuvent constituer un frein à la modification de leurs représentations sur l'ETP. Leur pratique de l'ETP n'étant pas importante (en terme d'inscription dans le temps ou de participations multiples), il n’y aurait pas eu de modification de cet objet de représentation car ce serait l'inscription progressive dans le programme (l'expérience répétée et durable) qui serait en mesure de transformer les représentations préexistantes sur l'ETP.

Les discours autour de la maladie et ses composantes ne sont pas non plus liés à la participation des patients au programme d'ETP. La littérature souligne que le rapport aux objets représentés se régulent au travers des pratiques mais également par la connaissance produite sur les objets représentés et par le niveau d'implication vis-à-vis de ces objets [37, 38]. Les représentations sur le diabète et ses composantes (e.g., règles hygiéno-diététiques, traitements médicaux...) ne peuvent alors évoluer que sous certaines conditions. La modification des contenus représentationnels nécessite l'activation prolongée et significative de nouvelles pratiques vis-à-vis du diabète, une consolidation progressive et stabilisée des connaissances, comme un niveau d'implication personnelle vis-à-vis du diabète et de sa gestion. L'unique participation à un programme d'ETP ne permettrait pas d'offrir ses conditions de changement pour moduler les représentations des patients sur la maladie et les soins.

Toutefois, la description de notre échantillon souligne que les patients en activité professionnelle n'ont pas participé au programme d'ETP et que ce sont majoritairement les patients retraités qui y ont participé. L'analyse Alceste souligne que ceux qui parlent le plus 
des objectifs de l'ETP sont les patients en activité professionnelle tandis que ceux qui parlent le plus du groupe sont les patients retraités. La participation au programme conduirait les patients retraités à parler davantage de l'expérience groupale auxquels ils ont participé plus que des objectifs poursuivis par le programme d'ETP, sur lesquels les patients actifs portent leurs discours. Il semble que l'expérience vécue du programme vienne renforcer un contenu existant de la représentation de l'ETP chez les patients retraités. Si on prend en compte le lien entre expérience vécue et représentations, on peut faire l'hypothèse que l'expérience du programme régule l'expression des contenus représentationnels de l'ETP tout en étant modérée par les variables socioéconomiques. Les échanges entre patients et professionnels deviennent des contenus plus saillants de la représentation de l'ETP pour les patients retraités ayant participé au programme. Enfin, les patients les moins aisées (e.g., inactifs ou au foyer) sont ceux qui n'ont pas participé à l'ETP (Cf. Tableau II). L'absence de relation entre les discours sur l'ETP, la non-participation à l'ETP et les autres variables socioéconomiques pourraient s'expliquer par une faible connaissance du programme et une faible implication vis-à-vis de celui-ci. Ce faisant ces patients ont une représentation de l'ETP moins marquée que les autres sur des contenus représentationnels spécifiques tels que les objectifs et le groupe en ETP. L'ensemble de ces constats soulignent l'importance des variables socioéconomiques sur la représentation que les patients possèdent de l'ETP (vécues ou pensées).

Pour conclure sur ce point, il nous semble important de discuter les « fondements » sociosymboliques structurants potentiellement la mise en œuvre des programmes d'ETP. Nous avons montré que les patients qui sont issus de milieux aisés s'inscrivent dans une démarche de prévention de leur santé et semblent davantage acteur de la prise en charge de leur maladie comparativement aux autres patients. Ces attitudes et comportements de santé et de maladie s'inscrivent dans la même « culture » médicale (centrée sur la prévention et l'autonomie) qui 
est dispensée dans les programmes d'ETP. Les programmes d'éducation thérapeutique reposent sur l'apport de connaissances portant sur l'équilibre alimentaire, la pratique d'une activité physique, la gestion du stress. Le rapport au corps et les règles hygiéno-diététiques proposés dans les programmes d'ETP s'inscrivent dans une «culture médicale » proche des recommandations auxquelles les patients aisés adhérents dans leur quotidien bien avant la survenue de leur maladie chronique. Nous pouvons nous demander, dans quelle mesure, les patients qui sont issus des milieux les moins aisés, peuvent «s'approprier » cette « culture » de gestion de la maladie véhiculée dans les programmes d'ETP puisqu'elle s'éloigne, pour partie, de leur «culture» (démarche curative et plus passive). En d'autres termes les programmes d'ETP favorisent-ils les représentations et comportements des patients issus de milieux aisés en étant plus proches de leur façon de penser et d'agir sur leur santé et leur maladie ? Ce constat peut conduire à s'interroger sur les processus de changements (des représentations et des comportements associés) que peut produire la participation à un programme d'ETP. Ces programmes sont-ils en mesure de modifier les contenus d'expériences et de représentations ou renforcent-ils, dans certaines conditions, les représentations et comportements préexistants chez les patients sur leur maladie, les soins et l'ETP ? Le lien entre les variables relevant des caractéristiques socioéconomiques et certaines dimensions spécifiques de la maladie et de l'ETP tend à illustrer le rôle important de l'inscription sociale initiale des patients. Il met également en évidence que les variables socioéconomiques ont un rôle sur les différentes dimensions relevant de la prise charge de la maladie par les patients : le choix du recours à l'ETP, l'acquisition des connaissances (en fonction des connaissances préexistantes) et de savoirs faires liés à la maladie (pratiques/comportements curatifs versus préventifs), et la forme d'implication (actif versus passif) dans la gestion de la maladie. 
Cette recherche met en évidence que « les caractéristiques du sujet et de l'objet ont une incidence sur ce qu'est la représentation » [39] et souligne l'importance du contexte social dans la construction de ces objets. L'ETP, par la façon dont elle prend forme dans l'univers médical et par la manière dont elle est proposée et mise en œuvre (démarche préventif et d'autonomie), semble renforcer l'impact du contexte social en favorisant les représentations et les comportements des patients les plus aisés au détriment des plus vulnérables. L'ETP est soumise à la même problématique que l'éducation à la santé, elle « se fait presque davantage au bénéfice des catégories aisées plutôt que modestes »[40] ou des patients qui en ont «le moins besoin » [41]. Une attention particulière est donc à porter sur l'offre d'ETP, afin qu'elle ne contribue pas au renforcement des inégalités sociales de santé plutôt que de les contrôler, voire de les atténuer.

\section{Conclusion}

Les résultats de cette recherche témoignent, à divers degrés, des enjeux de la nécessaire contextualisation de l'expérience de la maladie et des dispositifs de soins, de la prise en charge et de l'accompagnement de la maladie pour circonscrire les enjeux de l'ETP [12, 42, 43]. L'inscription sociale des patients/malades constitue une grille de lecture a priori des savoirs transmis au cours de la prise en charge et des dispositifs éducatifs comme l'ETP. Cette inscription sociale et les modes de rapport qu'elle introduit à la maladie conduisent à la valorisation sélective des éléments de représentations et d'expérience de la maladie qui semblent les plus pertinents au regard de cette inscription, par exemple, la valorisation de règles de hygiéno-diététiques chez les patients aisés. Par le biais de son inscription et de sa participation sociale, le patient/malade voit son «patrimoine de dispositions et compétences soumis à des forces différentes » [43]. Ces dispositions et compétences peuvent être appréhendées comme le produit de l'interaction entre des rapports de forces internes (dispositions incorporées à croire, voir, penser, agir) et externes (rôle des circonstances et 
contextes sociaux). Les représentations que les patients/malades produisent sur leur expérience et parcours de maladie, sur les soins et les dispositifs éducatifs permettent, selon nous, de dévoiler pour partie ce patrimoine de dispositions et compétences et témoignent de la dépendance des savoirs par rapport aux positions occupées par les acteurs, à leurs intérêts et leurs buts [44]. En d'autres termes, le contexte social des patients s'associe à un ensemble de valeurs et de normes en santé qui ont un impact sur la manière dont ils vont penser et agir visà-vis de leur maladie. Cette étude souligne la nécessité de considérer - le contexte social et les croyances sur la maladie qui y sont associés - dans la démarche d'ETP afin de favoriser la participation des patients les plus vulnérables aux programmes, d'intervenir de manière ciblée et adaptée sur les problématiques qui les concernent, d'améliorer leur prise en charge et contribuer in fine à une réduction des inégalités sociales de santé.

Conflits d'intérêts : aucun.

\section{Financement :}

Cette étude a bénéficié d'un financement de l'Agence Régionale de Santé de Provence Alpe Cote d'Azur (ARS-PACA) dans le cadre des actions innovantes. 


\section{Références}

1. Böhme P, Durain-Sieffert D, Fay R, Contal I, Floriot M, Chery B. Efficacité à court terme d'un réseau de ville centré sur l'éducation thérapeutique du patient diabétique de type 2 : comparaison avec un service hospitalo-universitaire. Diabetes Metab 2010; 36:A60.

2. Chevalier N, Dubus H, Galand-Portier MB, Meyer P. Intérêt de la personnalisation du projet d'éducation thérapeutique dans la prise en charge du patient diabétique de type 2 . Diabetes Metab 2008; 34:A104.

3. Bosquet F, Cosker K, Jacqueminet S, Nicolas N, Granger B, Hartemann A. Diabète de type 2 (DT2) : évaluation d'un programme d'évaluation thérapeutique en hôpital de jour. Diabetes Metab 2014; 40:A43.

4. Senez B, Felicioli P, Moreau A. Évaluation de la qualité de vie des patients diabétiques de type 2 en médecine générale. Presse Med 2004; 33:161-6.

5. Penfornis A. Observance médicamenteuse dans le diabète de type 2 : influence des modalités du traitement médicamenteux et conséquences sur son efficacité. Diab and Metab 2003; 29:31-37.

6. Vannereau D, Jaffiol C, Fontbonne A, Passeron S. Conséquences de la précarité sur le mode de vie et l'alimentation d'une population diabétique et pré-diabétique : à propos d'une enquête en Languedoc. Med Mal Métab 2014; 8:88-94.

7. Barnichon C, Ruivard M, Philippe $\mathrm{P}$, Vidal $\mathrm{P}$, Teissonière $\mathrm{M}$. Diabète de type 2 et précarité : une étude cas-témoins. Rev Med Int 2011; 32:467-471. 
8. Janand-Delenne B, Sejil S, Rocher L, Calemczuk G. Précarité, complications et niveau de connaissances sur le diabète dans une population de 125 patients. Diab and Metab 2010; 36:A45.

9. Sultan S, Hartemann-Heurtier A, Grimaldi A. Comprendre les patients pour promouvoir l'autorégulation dans le diabète de type 2 : Vivre avec une maladie évolutive qui commence avant son début. Diabetes Metab 2003; 29:21-30.

10. Pezé V, Dany L, Lumediluna ML, Thill JC. Rôle et impact de la situation de précarité sur l'expérience de la maladie des patients atteints de diabète de type 2. Med Mal Metab 2015; 9:523-528.

11. Jodelet D. La place des représentations sociales dans l'éducation thérapeutique. Éducation Permanente 2013; 195:37-46.

12. Fonte D, Apostolidis T, Lagouanelle-Simeoni MC. Compétences psychosociales et éducation thérapeutique du patient diabétique de type 1 : une revue de littérature. Santé Publique 2015; 26:763-777.

13. Jodelet D. Place de l'expérience vécue dans les processus de formation des représentations sociales. In : Les savoirs du quotidien. Transmission, appropriations, représentations. Rennes: Presses Universitaires de Rennes; 2006, p. 235-255.

14. Reinert M. Quelques interrogations à propos de l'« objet» d'une analyse de discours de type statistique et de la réponse « Alceste ». Langage et Société 1999; 90:57-70.

15. Kalampalikis N. L'apport de la méthode Alceste dans l'analyse des représentations sociales. In : Méthodes d'étude des représentations sociales. Ramonville Saint-Agne: Erès; 2003, p. 147-163. 
16. Doise W. L'ancrage dans les études sur les représentations sociales. Bull Psychol 1992; 45:189-195.

17. Caillavet F, Darmon N, Lhuissier A, Reignier F. L'alimentation des populations défavorisées en France : synthèse des travaux dans les domaines économique, sociologique et nutritionnel. Les travaux de l'Observatoire national de la pauvreté et de l'exclusion sociale, 2005-2006. Paris: La Documentation Française; 2006, p. 279-322.

18. Barrett JE, Plotnikoff RC, Courneya KS, Raine KD. Physical activity and type 2 diabetes: exploring the role of gender and income. Diab Educ 2007; 33:128-143.

19. Mormiche P. Pratiques culturelles, profession et consommation médicale. Economie et statistique $1986 ; 189: 39-50$.

20. Boltanski L. Les usages sociaux du corps. Annales ESC 1971, 26:205-33.

21. Fainzang S. Médicaments et société : le patient, le médecin et l'ordonnance. Paris : Presses Universitaires de France; 2001, p. 120-132.

22. Darmon N, Carlin G. Alimentation et inégalités sociale de santé en France. Cahiers de nutrition et de diététique $2013 ; 48: 233-239$.

23. Poulain JP, Laurence T. Alimentation et précarité : considérer la pluralité des situations. Anthropology of food [en ligne] 2008, 6. URL: http://aof.revues.org/4773

24. Perrot P. Le travail des apparences ou les transformations du corps féminin, XVIIe-XIXe siècle. Paris: Seuil; 1984, p.280.

25. Régnier F, Masullo A. L'impact des messages de santé liés à l'alimentation, rapport de recherche INRA-corela; 2007, p.180.

26. Régnier F., Masullo., 2009 « Obésité, goûts et consommation. Intégration des normes d'alimentation et appartenance sociale », Revue française de sociologie, 4, 50, p. 747-773. 
27. Lhuissier A., Régnier F., 2005 «Obésité et alimentation dans les catégories populaire : une approche de corps féminin », Inra sciences sociales, 3-4.

28. Schwartz O., 1990 - Le monde privé des ouvriers, Paris, Presses Universitaires de France.

29. Paugman S., 1993- La société française et ses pauvres, Paris, Presses Universitaire de France.

30. Régnier F., Lhuissier A., Gojard S., 2006 - Sociologie de l'alimentation, Paris, La Découverte. p70.

31. Vannereau D, Jaffiol C, Fontbonne A, Olive J-P, Passeron S. Conséquence de la précarité sur le mode de vie et l'alimentation d'une population diabétique et pré-diabétique : à propos d'une enquête en Languedoc. Med Mal Metab 2014; 8:88-94.

32. Reach. G. Insulinorésistance psychologique. Med Mal Metab 2008; 2:176-178.

33. Snoek F. Résistance psychologique à l'insuline : Quelles sont les principales craintes des patients et des médecins ? Diab Voice 2001; 46:26-28.

34. Raccah D, Molines L, Darmon P. Réalité actuelle en diabétologie. Les idées reçues. Les barrières à l'insulinothérapie. Med Mal Metab 2011; 5:86-88.

35. Abric JC. Pratiques sociales et représentations. Paris: Presses Universitaires de France; 2011, p. 312.

36. Guimelli, C. Transformation des représentations sociales, pratiques nouvelles et schèmes cognitifs de base. In Structures et transformations des représentations sociales. Lausanne: Delachaux et Niestlé; 1994, p.171-198.

37. Dany L, Abric JC. Distance à l'objet et représentations du cannabis. International Review of Social Psychology 2007; 20:77-104.

38. Dany L. From the study of social practices to the study of "distance to the object". Papers on Social Representations 2016; 25: 6.1-6.19. 
39. Jodelet D. Les représentations sociales. Paris: Presses universitaires; 1989, p. 454.

40. Fassin D, Grandjean H, Kaminski M, Lang T, Leclerc A. Les inégalités sociales de santé. Paris: La Découverte « Recherches »; 2000, p. 448.

41. Haut Conseil de la Santé Publique (2016). Avis relatif à la mise en œuvre de l'éducation thérapeutique du patient depuis la loi n²009-879 du 21 juillet 2009 portant réforme de l'hôpital et relative aux patients, à la santé et aux territoires (HPST).

42. Apostolidis T, Dany L. Pensée sociale et risques dans le domaine de la santé : le regard des représentations sociales. Psychologie Française 2012; 57:67-81.

43. Morin M, Apostolidis T. Contexte social et santé. Traité de psychologie de la santé. Paris: Dunod; 2002, p. 463-489.

44. Lahire B. Pour la sociologie. Paris: La Découverte; 2016, p. 184. 